\title{
Development of a test bench for vibratory fatigue experiments of a cantilever beam with an electrodynamic shaker
}

\author{
Aymeric Appert ${ }^{1, *}$, Christophe Gautrelet $^{1}$, Leila Khalij ${ }^{1}$, and Renata Troian ${ }^{1}$ \\ ${ }^{1}$ Normandie Université, LMN/INSA Rouen Normandie, 76000 Rouen, France
}

\begin{abstract}
When fatigue tests are carried out using a shaker, dynamic phenomena often appear and lead to a non-linear behaviour in the experiment. In this paper, the dynamic response of the specimen is studied in order to evaluate the impact on the results. The experiment consists in a notched beam fixed on the armature of an electro-dynamic shaker. The transfer function between the center of the armature and the tip of the beam is obtained with a swept sine near the first resonance frequency of the specimen. The drive is a constant acceleration amplitude. An evolution in the transfer function of the beam is observed when the drive is modified: the resonance frequency decreases and the damping increases. This non-linearity is investigated by studying the movement of the shaker, the fixture and the beam. The results show that the beam close to its resonance disrupt the imposed movement. An experimental setup correcting those defects is proposed.
\end{abstract}

\section{Introduction}

Conventional fatigue tests performed with servohydraulic testing machines can be costly and timeconsuming for high-cycle fatigue studies. One way to accelerate the tests is to carry out vibration testing with an electro-dynamic shaker. Different configurations of this experimental setup can be found in the literature.

The simplest method is to fix the specimen directly on the shaker armature by means of a clamp. Different shapes can be used for the specimen such as plate, beam or other complex designs to study multiaxial loading. In all cases, the fixture consists in constraining the sample between two parts tightened with multiple screws. The screws are placed either close to the embedding or going through the specimen. Generally, the choices made for the fixture are not explained in the literature.

Abdullah [1] fixed multiple beams on the shaker's table with 4 screws through the upper blocks and the specimen. He observed that damping increases with the augmentation of specimen tip amplitude and explained it by air damping. A similar fixture is used by Angeli et al. [2], Ge et al. [3] and Zhou et al. [3]. The specimens are fixed with 2 through-the-thickness screws. A groove is added in the clamp by Ge et al. and Zhou et al. to prevent the specimen rotation. Hooreweder et al. [5] used this specificity to ensure accurate contact surfaces. In [6], George et al. maintained the plate-shaped specimen between two blocks with two screws positioned close to the embedded part. One objective was to reduce the potential damping in the clamp, that's why the maximum torque was applied to the mounting bolts. Habtour et al. [7] precise that non-linearity may appear due to the clamping but assume that it is negligible. No more precisions are given on the used fixture as in Hammami et al. [8], Paulus et al. [9] or Li et al. [10]. Only pictures of the experiments show the fixture used. Ellyson et al. [11] don't even show the clamping but precise that their results exhibit localized slipping at the clamped edge. The importance of the clamping strategy is highlighted. Rognon [12] and Zanellati et al. [13] used rod-shaped specimens, but the clamping was still made of multiple parts tightening the beam. Rognon added a flat part to the beam to ensure a good positioning of the specimen and control the tightening torque.

The literature points out the importance of the clamping. In vibration testing, this part has a huge impact on the dynamic response and modal parameters are directly used in fatigue tests to predict the damage $[14,15$, 16]. However, we have seen that phenomena induced by the fixture are often neglected or taken into account as a structural damping. In this work, we have highlighted the importance of the fixture design for accurately assessing the modal parameters.

The first part of this paper presents an experimental setup to carry out fatigue tests on a beam with an electrodynamic shaker. The behaviour near the first resonance frequency is studied by doing swept sines at different excitation levels. The results reveal unexpected non-linearities in the structure and the causes are investigated. The second part shows the study achieved in order to eliminate non-linearities and suggests improvements in the experimental setup. Those improvements are tested in the third part and the results are discussed.

\footnotetext{
${ }^{*}$ Corresponding author: aymeric.appert $@$,insa-rouen.fr
} 


\section{Experimental setup}

\subsection{Equipment}

The test rig in Fig. 1 consists in an air-cooled electrodynamic shaker driven by an acquisition control peripheral (ACP) with the help of a power amplifier. The ACP is used to control the shaker in a closed loop. Thus, an acceleration, velocity or displacement profile is applied on a localised point of the structure instead of an excitation force.

The specimen is fixed directly on the armature of the shaker, clamped between two rectangular plates by means of four screws at the corners. The screws are tightened with the same torque. A wedge is added between the two plates to force them to remain parallel and ensure a good contact surface on the beam.

The driving point is an accelerometer placed in the center of the clamp, on the axis of the shaker. The transmissibility function is obtained thanks to a vibrometer measuring the velocity at the tip of the beam. In this paper, only swept sines are used, thus the acceleration is easily obtained from this velocity. A vibrometer was preferred to an accelerometer on this location, because the mass and rigidity of the accelerometer wire have a significant effect on the beam dynamic behaviour [17].

\subsection{Beam design}

The shape of the beam was determined by carrying out a modal analysis with Code_Aster. The objective is to find the resonance frequencies and the associated modal shapes of the sample. The modal shape gives the type of loading the area of interest is subjected to. In our case, we use the first flexural mode of vibration of a steel beam. Another point to consider about the geometry is that the maximum stress will be located in the fixture. The crack cannot be studied in this area. The most common way to avoid this problem is to create a stress concentration by adding a section reduction far from the clamp [18].

The mesh visible in Fig. $\mathbf{2}$ is made of quadrangular plate elements based on the Kirchhoff theory, to avoid shear locking. The properties used for the simulation are given in Table 1.

Table 1. Mechanical properties of the steel specimen

\begin{tabular}{|c|c|c|c|}
\hline $\mathrm{E}(\mathrm{GPa})$ & $v$ & $\rho\left(\mathrm{kg} / \mathrm{m}^{3}\right)$ & $h(\mathrm{~mm})$ \\
\hline 205 & 0.3 & 7696 & 1 \\
\hline
\end{tabular}

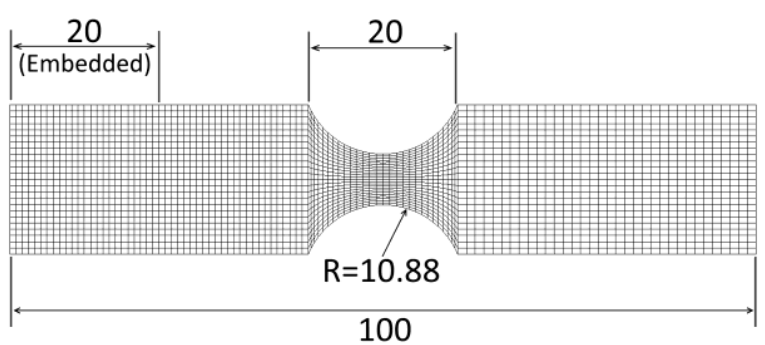

Fig. 2. Mesh and dimensions of the beam ( $\mathrm{mm})$

The first natural frequency at $114.12 \mathrm{~Hz}$ corresponds to the first flexural mode of the beam. This mode is far from the second one which is also a flexion at $810.63 \mathrm{~Hz}$. The maximum stress is not located close to the fixture thanks to the section reduction (Fig. 3). Thus, the fracture will occur in this stress concentration zone and will be easy to detect. In comparison, the second mode has relatively high stresses close to the fixture. This configuration can't be used for fatigue tests because the fracture location is not well controlled.

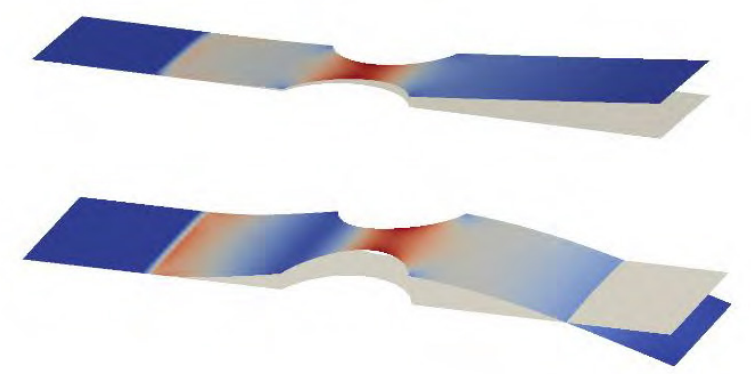

Fig. 3. Modal shapes of mode at $114.12 \mathrm{~Hz}$ (above) and 810.63 $\mathrm{Hz}$ (below) with stress repartition.

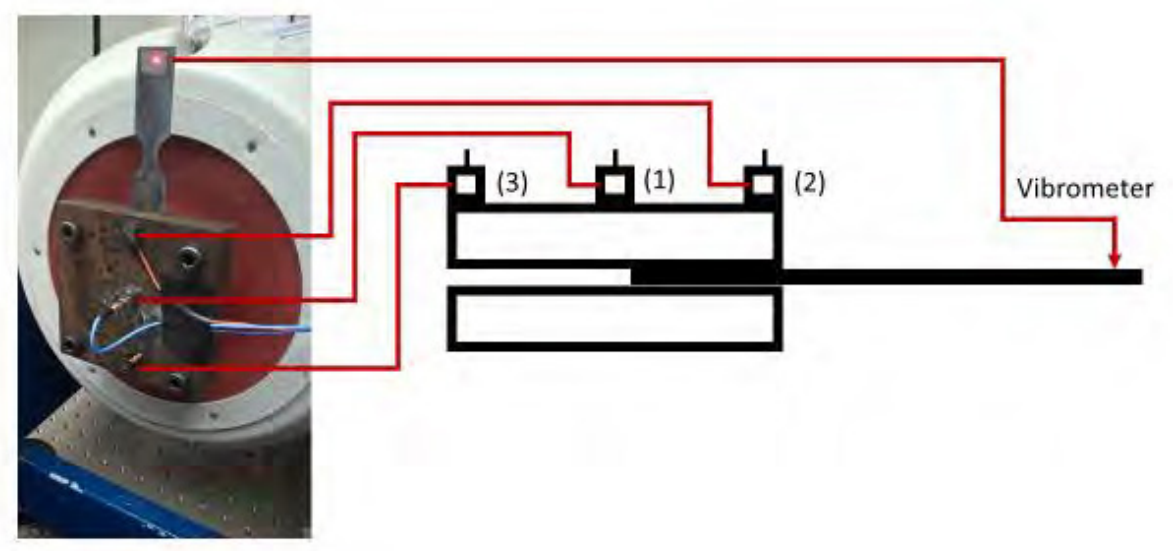

Fig. 1. The experimental setup 


\subsection{Experimental test}

The tests performed are swept sines at different levels of acceleration, from $0.5 \mathrm{~g}$ to $1 \mathrm{~g}$ every $0.1 \mathrm{~g}$, in a frequency range surrounding the first resonance $(100 \mathrm{~Hz}-$ $120 \mathrm{~Hz}$ ). The sweep speed is $0.1667 \mathrm{~Hz} / \mathrm{sec}$. The control is carried out by a triaxial accelerometer (noted (1) on Fig. 1), which is placed in the center of the fixture. Obviously, only the acceleration in the axis of the shaker is used for the drive, but we also measure the transversal accelerations. Two other accelerometers are fixed on the clamp, one where the specimen is fixed (noted (2)) and one at the opposite (noted (3)), symmetrically to the shaker axis.

\subsection{Results and discussion}

The transmissibilities plotted in Fig. 4 reveal nonlinearities in the experiment and a few hypotheses can be made about their sources. The resonance frequency is shifting to the left and the damping increases with the excitation level. The tests were made at very low acceleration levels to ensure that there is no geometrical non-linearity nor plastification of the beam. Moreover, after all the swept sines realized at growing accelerations, a last sweep was carried out at the smallest level. The transmissibility between the first and the last test at the same excitation were identical which reject any plastic strain hypothesis.

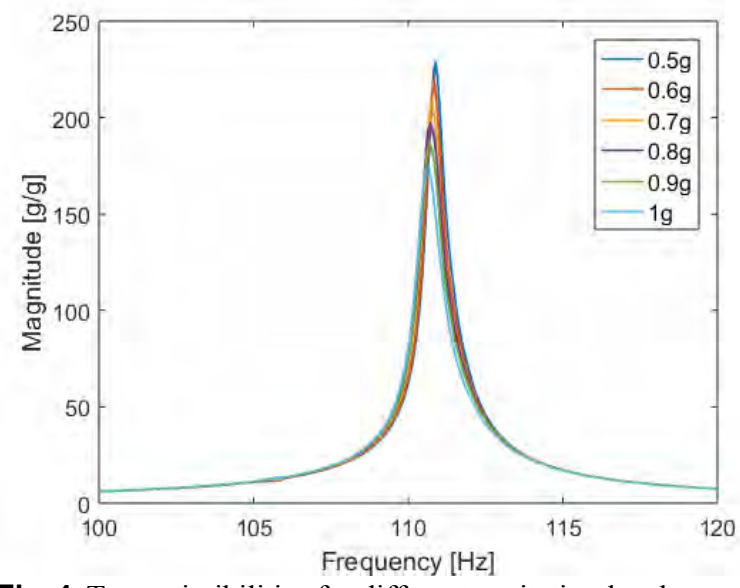

Fig. 4. Transmissibilities for different excitation levels

The accelerometers (2) and (3) placed on the fixture reveals a spurious displacement of the shaker when the swept is close to the resonance of the beam. Fig. 5 represents the accelerations at those points for an excitation of $0.5 \mathrm{~g}$ and $1 \mathrm{~g}$. The difference between the acceleration given by the control accelerometer and the others reaches $30 \%$ of the driving acceleration.

The pattern of the curve tends to show that the specimen drives the armature in a rotation movement (see Fig. 6). Before the resonance frequency, the beam is amplifying the movement of the shaker on its side. Then the phase is shifting, and the rotation is in opposition to the shaker displacement. This shift is clearly visible with the accelerations measured by (2) and (3).

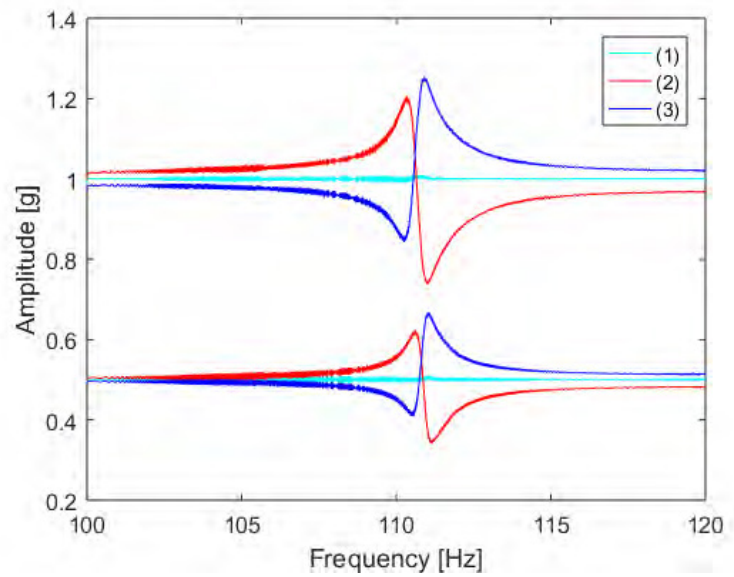

Fig. 5. Acceleration amplitudes measured by the three accelerometers for excitation levels of $0.5 \mathrm{~g}$ and $1 \mathrm{~g}$

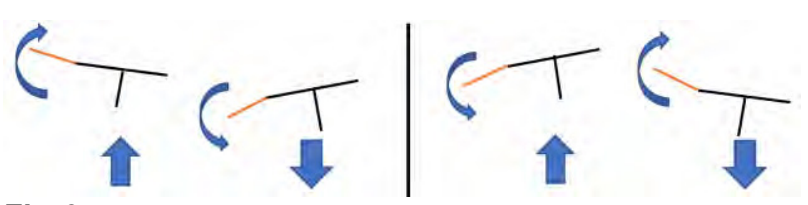

Fig. 6. Rotation movements induced by the specimen before and after the phase shifting.

With the triaxial accelerometer, a high acceleration amplitude is measured in the direction of the beam when the frequency is close to the resonance of the specimen (see Fig. 7). In the perpendicular direction, only noise can be measured. This observation suits with the hypothesis described above.

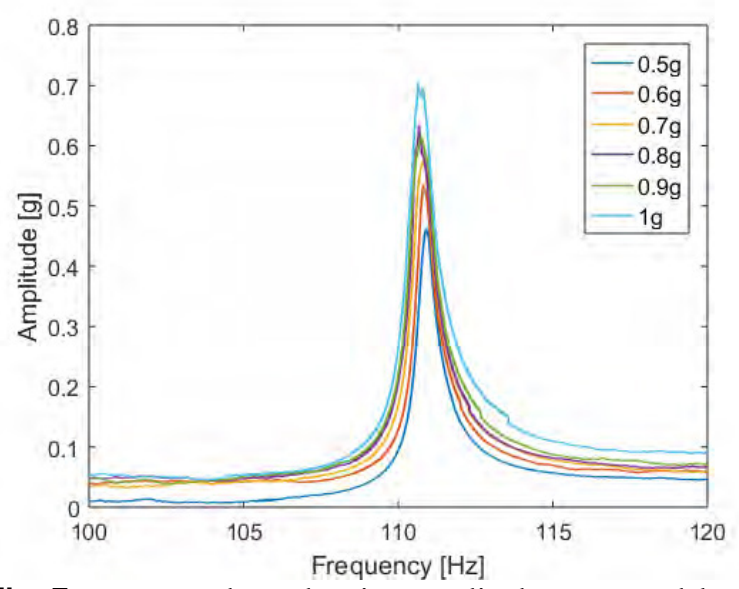

Fig. 7. Transversal acceleration amplitudes measured by the triaxial accelerometer (1) in the direction of the beam

One major issue with this phenomenon is that you can plot different transmissibilities with the same test, giving different modal parameters. This is illustrated in Fig. 8, where the accelerometers (1), (2) and (3) are used successively as the input for an excitation of $1 \mathrm{~g}$. Here we can find three resonance frequencies (respectively 110.6 $\mathrm{Hz}, 110.9 \mathrm{~Hz}$ and $110.5 \mathrm{~Hz}$ ) with three associated amplification (175.4, 196.6 and 175.7), which is not logical. There shouldn't be a choice for the input acceleration. 


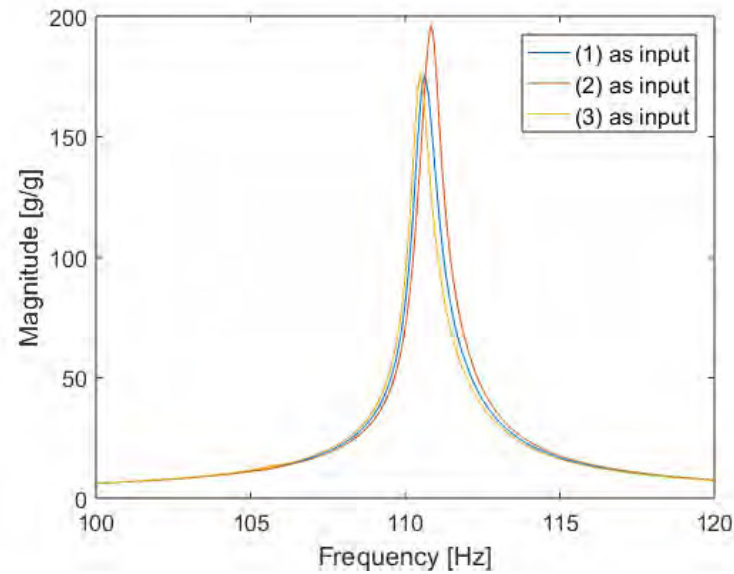

Fig. 8. Transmissibilities at $1 \mathrm{~g}$ with the three accelerometers as input acceleration

Another cause of this non-linearity could be the fixture. Without the wedge to ensure that the two plates remain parallel, the resonance frequency is lowered by more than $10 \mathrm{~Hz}$. In our case, the upper plate will inevitably bend under the pressure of the screws, such that the contact surface is located on the edges closest to them and not on the edge of the fixture. The free part of the specimen is not clearly dissociated and the resonance mode used depends strongly on the length of the beam. This deformation can be seen in the Fig. 9. The plates on the fixture have been used for high-cycle fatigue tests [19]. The successive mounting and unmounting of the specimens resulted in a permanent deformation of the upper plate. This picture shows clearly how the contact is located in the clamp, even with a thicker plate that will not be irreversibly deformed.

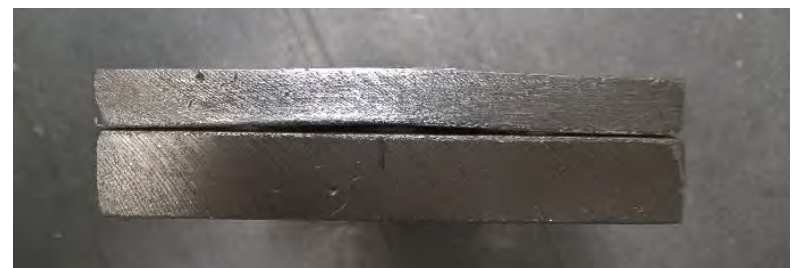

Fig. 9. Plates used as fixture for high-cycle fatigue tests

\subsection{Verification of the shaker}

The shaker was verified by carrying out the same sweeps as described in the previous part, but without the beam. The two plates were stilled fixed on the armature with the same tightening torque. The accelerations of the three accelerometers (1), (2) and (3) are plotted in Fig. 10 for each level of excitation. The transversal accelerations are negligible. The spurious displacements observed in the experiments of the previous section are completely caused by the beam resonance.

The mass of the fixture and the specimen is only 1.8 $\mathrm{kg}$. This is way below the shaker's capacity according to its documentation. We have observed the same phenomenon on other shakers.

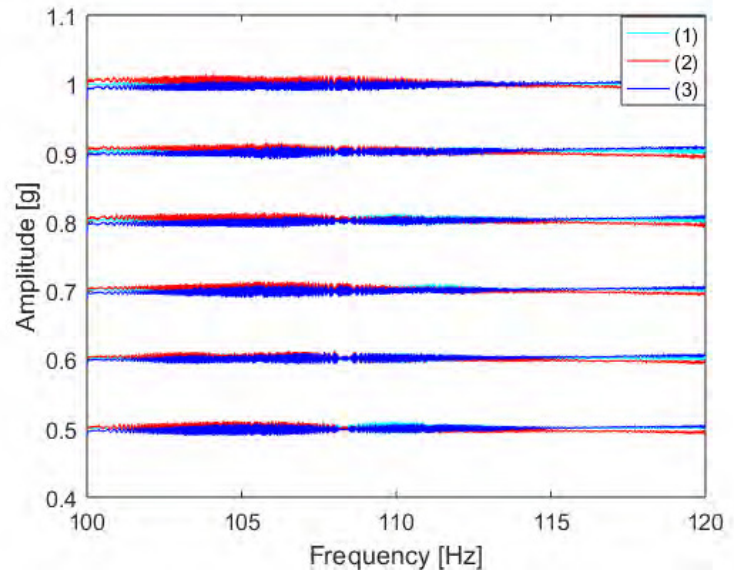

Fig. 10. Acceleration amplitudes measured by the three accelerometers without the beam

\section{Improvement of the test bench}

\subsection{Rotation of the armature}

Firstly, we tried to equilibrate the axis by placing two beams symmetrically on the armature, but we didn't observe any improvement. Indeed, the movement is disturbed because of the specimen resonance. Regarding the variabilities of the geometrical dimensions and of the positioning in the clamp, two beams cannot have exactly the same resonance frequency, and then the same dynamic response. As a consequence, the resonance of one specimen disrupted the resonance of the other and reciprocally.

A similar observation was noted by Rognon [12]. He carried out fatigue tests on an electrodynamic shaker with three specimens placed at $120^{\circ}$. This configuration was sufficient to reduce the transversal movements, but when a crack occurred on one of the beams, the modal parameters changed. It was then impossible to continue the test for the two other beams because of the change in the excitation.

Our proposal is to ensure a high rigidity in the direction of the beam. For that, we realised a mounting using roller bearings, separated from the shaker. A sketch presenting the setup is visible on Fig. 11.

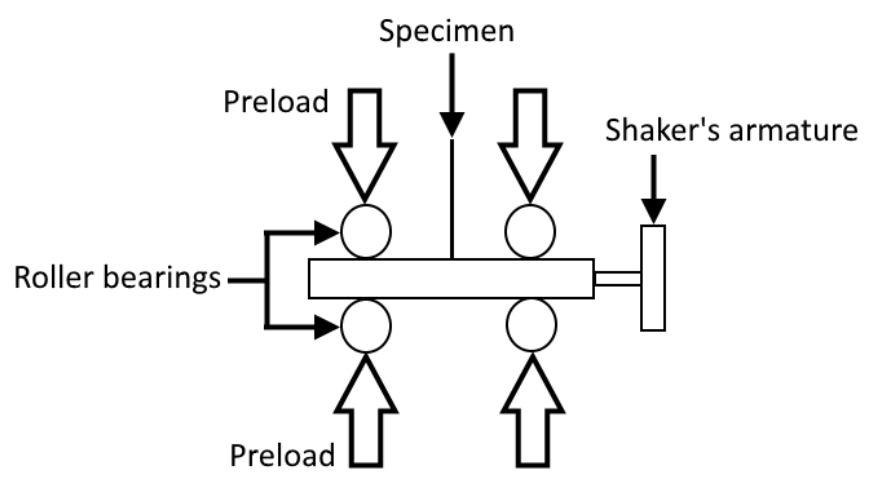

Fig. 11. New setup designed to avoid rotation of the fixture 


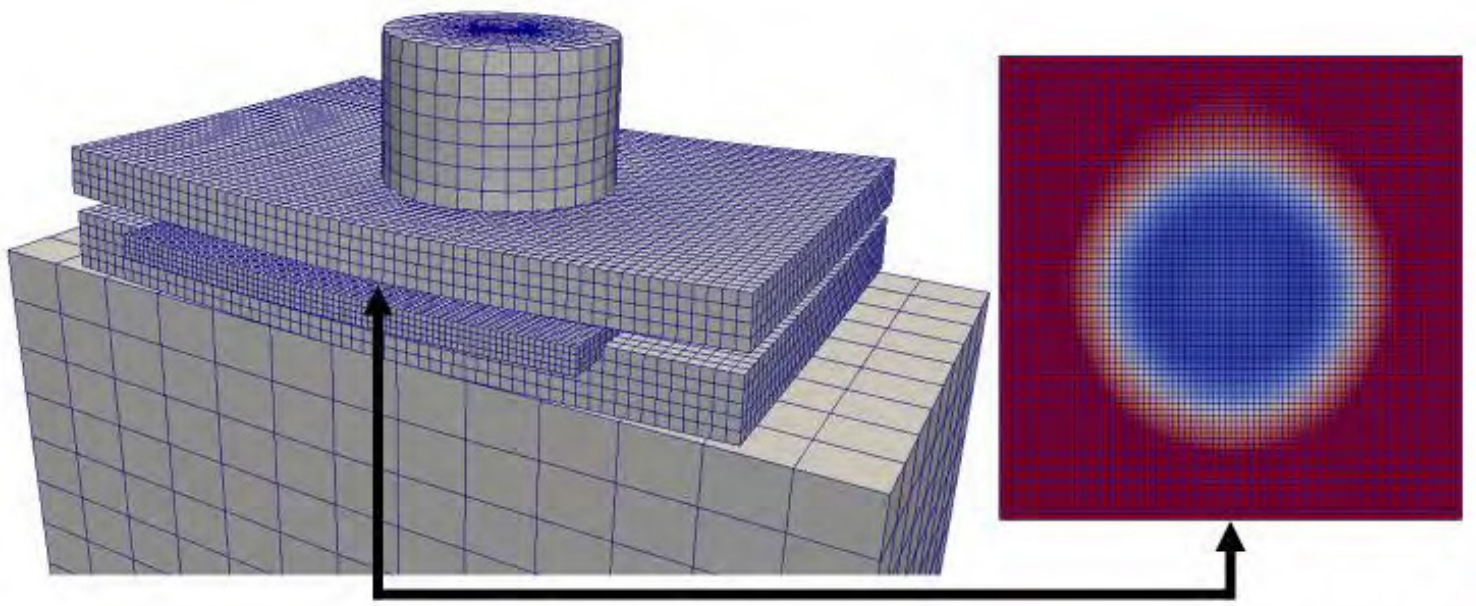

Fig. 12. Deformed shape and contact pressure between the upper plate and the beam. Red color (external area) corresponds to no pressure and blue color (internal area) to maximal pressure. The arrows indicate the same point on both views.

The preload and the high rigidity of the chosen roller bearings guarantee that not a rotational movement will appear. The shaker axis is not perturbed by the resonance of the sample. Another advantage is that it is possible to mount and unmount the specimen without submitting the shaker to tightening forces. Realizing such a setup is also less expensive than using an oversized electrodynamic shaker.

However, the preload will increase the required force to drive the experiment. The maximal attainable acceleration will be reduced because not only the mass of the moving part has to be considered. There is a dilemma between this maximal capacity of the shaker and the transverse rigidity.

\subsection{Contact surface in the clamp}

A non-linear quasi-static finite element analysis has been carried out in Code_Aster to study the contact in the fixture. The two objectives are:

- have a continuous contact along the edge of the fixture, where the beam is located.

- use only one screw for the tightening. Indeed, using multiple screw yields to a complicated mounting and affects repeatability. It's easier to control only one tightening torque.

A first study has been realized to see if it's possible to find a particular shape for the clamping parts. Fig. 13 presents a sketch of the simulation. The load is applied

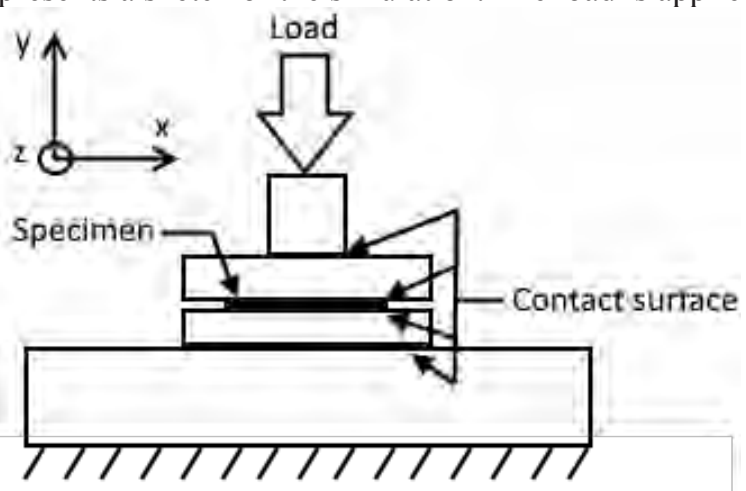

Fig. 13. Boundary conditions for the simulation in Code_Aster thanks to a cylinder which represents the screw. A displacement is imposed on its upper face. The specimen is sandwiched between two parts and the whole is pressed against a last part embedded at the opposite. This is useful to let the part under the sample deform freely. Contacts are considered without friction on each surface because displacements are very small. The surface of the cylinder where the load is applied is blocked in the transverse directions $\mathrm{x}$ and $\mathrm{y}$. The two plates and the specimens can't only be maintained by contact in Code_Aster, so springs with negligible stiffness have been added in the $x$ direction and they are clamped in the $\mathrm{z}$ direction on the faces where the beam is not emerging.

Fig. 12 shows the deformed shape with a scale factor of 100 and the contact pressure on the upper surface of the specimen. Almost the same contact pressure can be observed on the lower surface. It is clear that the specimen is clamped only close to the screw. The free length of the beam with such a fixture is not well defined. The conclusion was the same with other shapes for the two plates, with different thickness, angles, ...

Some setups can be found in the literature where the fixture is realized with cylinders. This kind of embedding can be used when the specimen is symmetric. If not, the vibration of the beam from one side of the cylinder will have an impact on the other side. This phenomenon is problematic in fatigue tests. Even if we consider that the specimen is perfectly symmetric at the beginning of the test, cracking will not appear symmetrically.

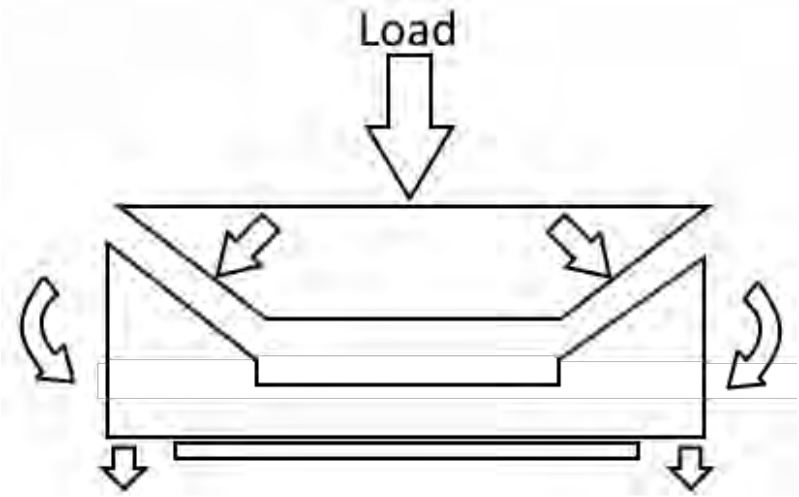

Fig. 14. Representation of the forces in the upper parts to ensure a good fixture 


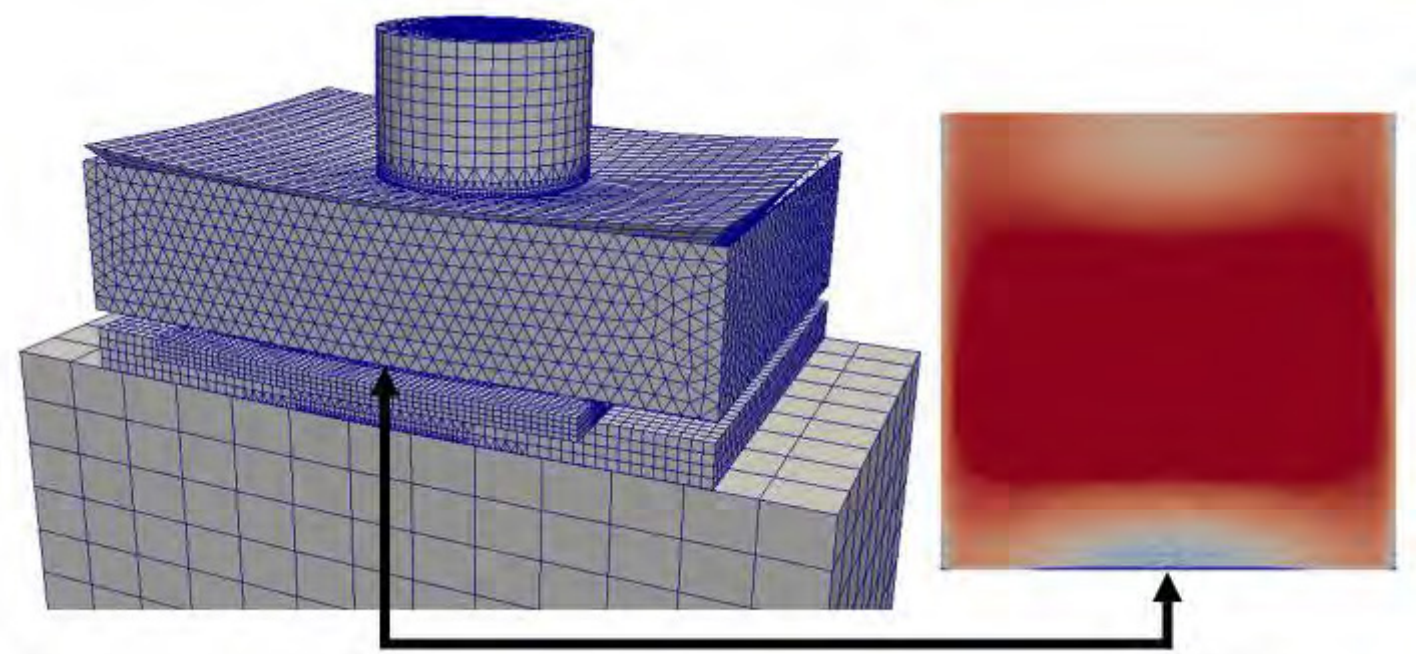

Fig. 15. Deformed shape and contact pressure between the upper piece and the beam. Red color (internal area) corresponds to no pressure and blue color (external area) to maximal pressure. The arrows indicate the same point on both views.

Finally, the solution found to this problem is to use two pieces instead of one for the upper plate. Fig. 14 explains the principle used. In this case, the contact pressure will be maximal on all the fixture edges of the specimen, determining clearly the limits of the embedding. The results of a new simulation with this geometry are shown on Fig. 15.

The pressure distribution is at its expected maximal where the beam goes out the fixture. On this side, the contact force is not constant along the edge. This is due to the shape of the embedding pieces: their width is bigger than their length. Using a square instead of this rectangle would give a more regular contact pressure, but we want to keep the possibility of using beam with variable width.

\section{New test bench}

\subsection{Experimental setup}

The test bench shown on Fig. 16 was created using the improvements detailed previously. Some attention was paid to the alignment and the preloading of the roller bearings.
The tests performed with the new setup are exactly the same as in part 2.3. The same specimen is used and accelerometers are placed such that equivalences can be found with the experiment in Fig. 1. The drive is controlled with accelerometer (1). The same acceleration is measured by the triaxial accelerometer (2) and compared. The rotational displacement observed with the previous experiment is measured by the accelerometers (2) and (3). Velocity is measured with the vibrometer at the same point.

\subsection{Results and discussion}

We can see on Fig. 17 that the fixture is not rotating because of the resonance of the beam. The curves correspond to the acceleration given by (2) in the direction of the specimen and (3) for an excitation of $1 \mathrm{~g}$. They can be compared to the previous setup with Fig. 7. There is still a perturbation around the resonance frequency, but it is highly reduced. With the first test rig, this transversal acceleration represented more than $70 \%$ of the driving acceleration. Now, the signal is relatively noisy. A better alignment should reduce the mean value observed but the impact of the beam resonance represents only a change in the amplitude of approximately $0.04 \mathrm{~g}$.

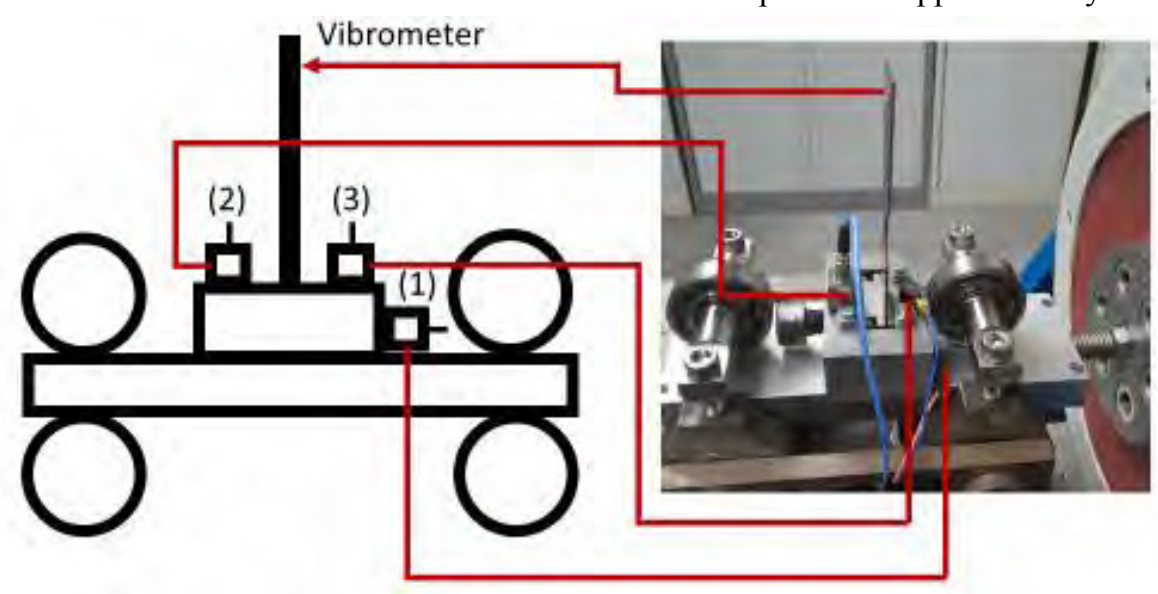

Fig. 16. Illustrative and real view of the setup 


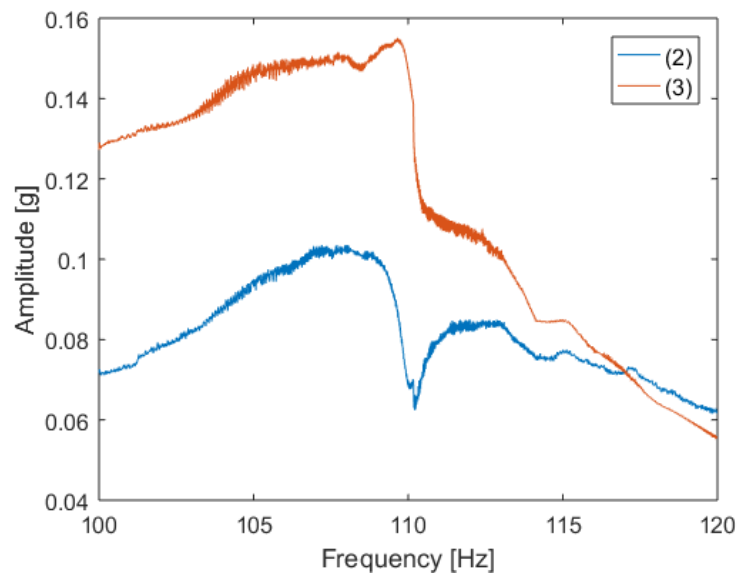

Fig. 17. Acceleration amplitudes in the beam direction given by accelerometer (2) and (3) for and excitation level of $1 \mathrm{~g}$.

This observation is confirmed if we compare the results of the driving accelerometer (1) and accelerometer (2) (see Fig. 18). They are placed on two opposite points on the fixture and not at the same height. If there is a rotation, we should see the same pattern as in Fig. 5. The two signals are identical, only a little peak of $0.01 \mathrm{~g}$ can be observed at the resonance, which is negligible. The transmissibility is not dependent from which accelerometer is chosen for the input.

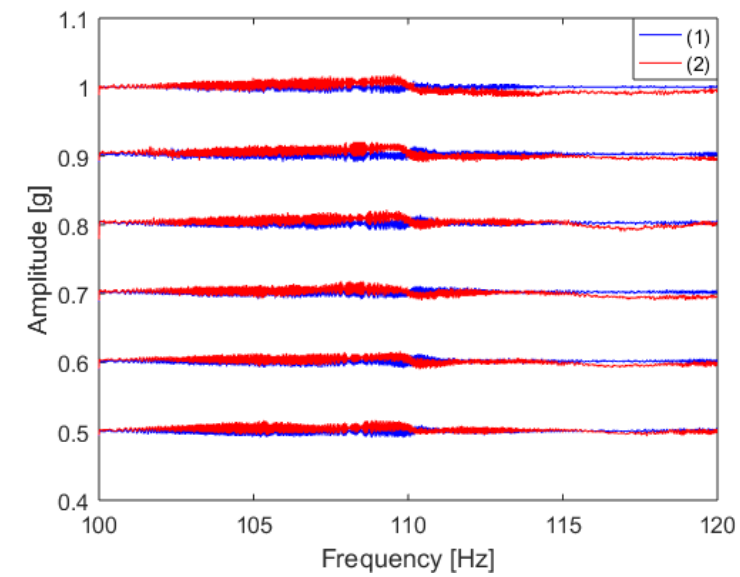

Fig. 18. Acceleration amplitudes given by the controlling accelerometer (1) and accelerometer (2) in the direction of the excitation.

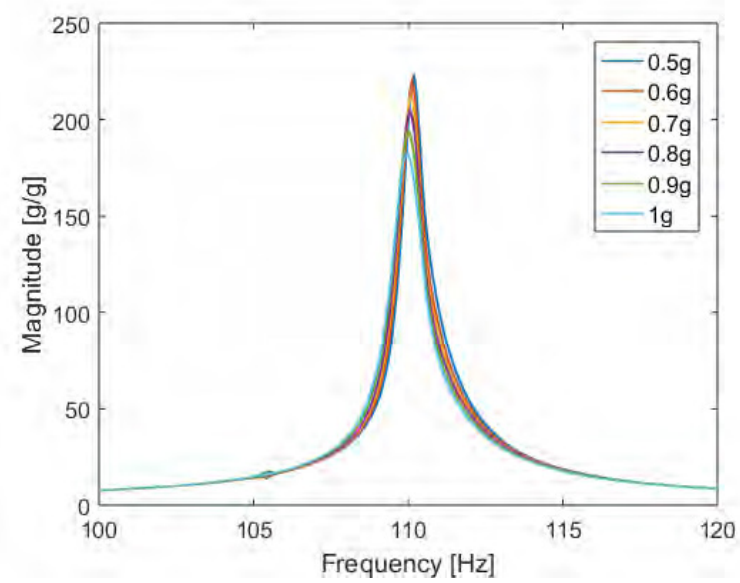

Fig. 19. Transmissibilities for different acceleration levels
Transmissibilities are plotted on Fig. 19. There is still a modification in the response when the excitation is changing. However, this effect is reduced. The increase in damping (Fig. 20) and the softening (Fig. 21) are less important than with the first setup.

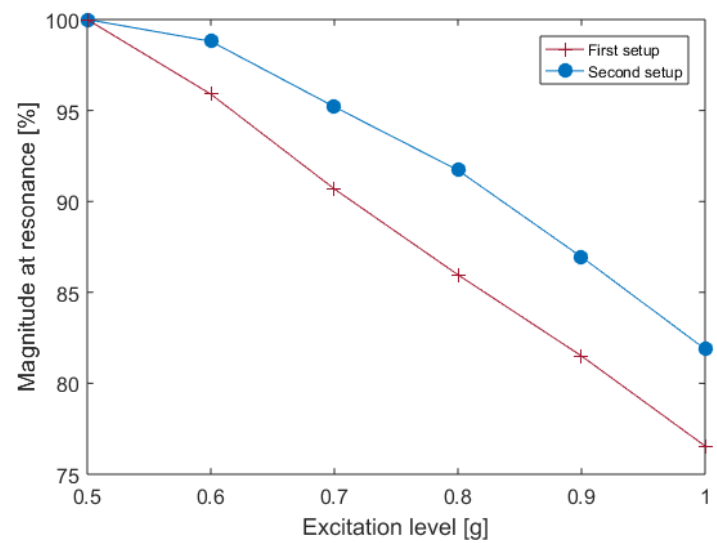

Fig. 20. Normalized magnitudes at resonance frequency for both setups according to the $0.5 \mathrm{~g}$ excitation level.

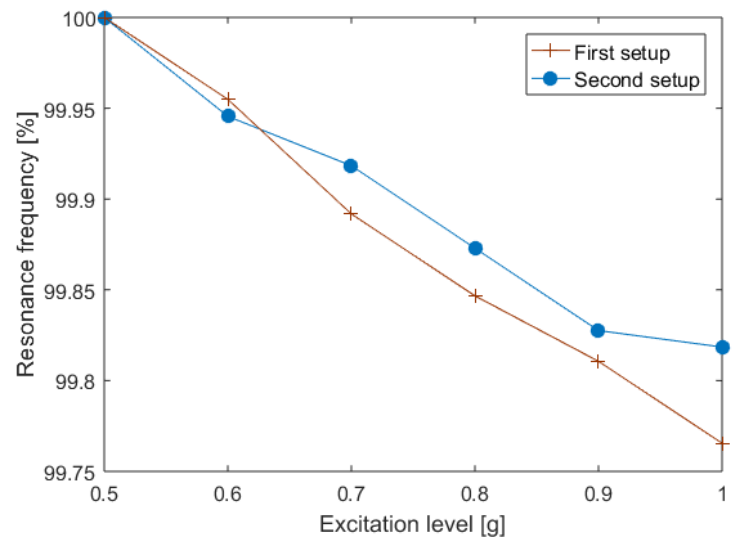

Fig. 21. Normalized resonance frequencies for both setups according to the $0.5 \mathrm{~g}$ excitation level.

The improvement in the results show that there are multiple causes for this non-linearity. Other hypotheses are still under study and will be presented in future works.

\section{Conclusion}

A test bench for vibratory fatigue experiments was developed. Non-linearities were observed with a classical setup for this kind of test. The fixture has a huge impact on the results and the specimen can distort the excitation movement. Solutions were investigated and taken into account for the design of a new test rig. The proposed fixture enables a better reproducibility in the experiment by ensuring a well-defined contact surface and an easy control of the tightening with only one screw. The transversal rigidity is also controlled and ensures a good excitation. However, the non-linearity was not completely eliminated and the study is still under consideration. 


\section{Acknowledgements}

The authors gratefully acknowledge the financial support provided by "La Région Normandie" and the European Union through the large-scale research network TERA (MRT MADNESS) - "Grands Réseaux de Recherche Territoire, Environnement, Risques, Agronomie" (Technological Risks Control Network).

\section{References}

1. A.B.M Abdullah, Development of a closed-loop resonant fatigue testing methodology and experimental life test of aluminum alloy, University of Akron (2010)

2. A. Angeli, B. Cornelis, M. Troncossi, Synthesis of Sine-on-Random vibration profiles for accelerated life tests based on fatigue damage spectrum equivalence, Mechanical Systems and Signal Processing 103, 340-351 (2018)

3. J. Ge, Y. Sun, S. Zhou, L. Zhang, Y. Zhang, Q. Zhang, A hybrid frequency-time domain method for predicting multiaxial fatigue life of 7075-T6 aluminium alloy under random loading, Fatigue Fract. Eng. Mater. Struct. 38, 247-256 (2015)

4. S. Zhou, Y. Sun, L. Guo, Random fatigue life prediction of carbon fiber-reinforced composite laminate based on hybrid time-frequency domain method, Random Advanced Composite Materials (2016)

5. B. Van Hooreweder, D. Moens, R. Boonen, P. Sas, Fatigue strength analysis of notched aluminium specimens using the highly stressed volume method, Fatigue Fract. Eng. Mater. Struct. 35, 154-159 (2011)

6. T. J. George, J. Seidt, M.-H. Herman Shen, T. Nicholas, C. J. Cross, Development of a novel vibration-based fatigue testing methodology, International Journal of Fatigue 26, 477-486 (2004)

7. E. Habtour, D. P. Cole, J. C. Riddick, V. Weiss, M. Robeson, R. Sridharan, A. Dasgupta, Detection of fatigue damage precursor using a nonlinear vibration approach, Struct. Control Health Monit. 23, 14421463 (2016)

8. M. Hammami, A. El Mahi, C. Karra, M. Haddar, Vibration non linéaire des composites stratifiés sains et endommagés par délaminage, CFA 2016 / VISHNO, 2435-2440 (2016)

9. M. Paulus, A. Dasgupta, E. Habtour, Life estimation model of a cantilevered beam subjected to complex random vibration, Fatigue Fract. Eng. Mater. Struct. 35, 1058-1070 (2012)

10. Y. Li, B. Sun, J. Fang, X.-F. Liu, T. Liang, G.-B. Cai, Fatigue Fract. Eng. Mater. Struct., 1-8 (2017)

11. B. Ellyson, M. Brochu, M. Brochu, Characterization of bending vibration fatigue of SLM fabricated Ti6Al-4V, International Journal of Fatigue 99, 25-34 (2017)
12. H. Rognon, Comportement en fatigue sous environnement vibratoire: Prise en compte de la plasticité au sein des méthodes spectrales, Ecole Centrale Paris (2013)

13. D. Zanellati, D. Benasciutti, R. Tovo, Vibration fatigue tests by tri-axis shaker: design of an innovative system for uncoupled bending/torsion loading, Procedia Structural Integrity 8, 92-101 (2018)

14. H. Sohn, C. R. Farrar, F. M. Hemez, D. D. Shunk, D. W. Stinemates, B. R. Nadler, J. J. Czarnecki, $A$ Review of Structural Health Monitoring Literature: 1996-2001 (2004)

15. O. S. Salawu, Detection of structural damage through changes in frequency: a review, Engineering Structures, Vol. 19, No. 9, 718-723 (1997)

16. A. C. Altunisik, F. Y. Okur, V. Kahya, Modal parameter identification and vibration based damage detection of a multiple cracked cantilever beam, Engineering Failure Analysis 79, 154-170 (2017)

17. J.-S. Dupuy, Identification des propriétés mécaniques de matériaux composites par analyse vibratoire, Université Montpellier II - Sciences et Techniques du Languedoc (2008)

18. T. Straub, Experimental Investigation of Crack Initiation (2016)

19. L. Khalij, C. Gautrelet, A. Guillet, Fatigue curves of a low carbon steel obtained from vibration experiments with an electrodynamic shaker, Materials and Design 86, 640-648 (2015) 